\title{
QUANTITATIVE MICROVASCULAR CHANGES DURING AZASERINE-INITIATED PANCREATIC CARCINOGENESIS*
}

\author{
KRIsZTina NaGy, Z. PÁlfia and G. RÉZ** \\ Department of General Zoology, Eötvös Loránd University, P.O. Box 330, H-1445 Budapest, Hungary
}

(Received: January 10, 2001; accepted: March 2, 2001)

\begin{abstract}
Although angiogenesis is considered to be indispensable for continuous tumour growth, only very few studies have been published performing microvessel quantification during tumour progression. We measured the tumour vascularity in different stages of rat pancreatic carcinogenesis induced by azaserine and promoted by raw soya flour-containing pancreatotrophic diet. Besides the tumour samples taken at 6 (atypical acinar cell nodules), 15 (adenomas) and 20 (localised adenocarcinomas) months after carcinogen initiation, we also investigated 3 control groups: tumour-bearing host tissue of azaserine-treated rats and normal tissue of untreated rats kept on standard or pancreatotrophic diet. In contrast with the usual microvessel counting on hot spots, we determined microvascular surface density $\left(\mathrm{S}_{\mathrm{V}}\right)$ and volume density $\left(\mathrm{V}_{\mathrm{V}}\right)$ by electron microscopic morphometry. There was no significant difference in these respect between the control groups. At month 6 after the azaserine induction $\mathrm{S}_{\mathrm{V}}$ and $\mathrm{V}_{\mathrm{V}}$ showed slight, nonsignificant decrease as compared to the host control. Both values remained unchanged until the 15th month and increased significantly by the 20th month. These results may indicate comparable growth rate of tumour and new microvessels in the premalignant stages of carcinogenesis while a more intense angiogenesis than tumour growth afterwards.
\end{abstract}

Keywords: Microvessel - quantitative - tumour progression - pancreas - rat

\section{INTRODUCTION}

Now it is generally accepted that angiogenesis, the development of new capillaries from pre-existing vessels, is essential for continuous tumour growth [6,9]. Although in the recent years, a large number of studies on quantification of the tumour vascularity have been published there are hardly any data about quantitative microvascular changes during tumour progression. The reason is that in most investigations the degree of vascularity was assessed in human tumours and in these cases the onset of carcinogenesis obviously could not be determined. Therefore, only groups of tumours with diverse histological structure or with benignous and malignous feno-

\footnotetext{
*Dedicated to Professor János Kovács on the occasion of his 70th birthday.

**Corresponding author; e-mail: grez@cerberus.elte.hu
} 
types are comparable and samples from different time points of tumour progression cannot be compared. There were some experiments in which tumour vascularity was quantitated in animal tumours but these studies are usually concentrated on the effect of various treatments and not on changes during the course of carcinogenesis. By means of the azaserine-rat model [22] we investigated the microvascular network morphometrically at different time points after the chemically-induced tumour progression, i.e. in different stages of pancreatic adenocarcinoma. These stages have been determined by Longnecker [11] according to the tumour size: the smallest neoplastic lesions that can be seen only under light microscope are called atypical acinar cell nodules (AACNs), the tumours of macroscopic size are named adenomas under $7 \mathrm{~mm}$ or adenocarcinomas above $7 \mathrm{~mm}$ in diameter.

The most frequently used method for quantitative characterisation of the tumour vascularity is based on immunohistochemical staining of the endothelial cells (usually with antibody against factor VIII-related antigen, CD31 or CD34) after which the number of microvessels per unit area (called microvessel count or microvessel density) is counted under light microscope in some areas of the most active neovascularization (so-called hot spots) $[4,8,21]$. This method has disadvantages: a) selection of the most vascularized areas are often subjective (except using computerassisted image analyser) and it may lead, as a consequence, to different results in case of experienced and inexperienced investigators [19] or between repeated counts by the same observer [3]; b) microvessel counts obtained by various antibodies are sometimes significantly different [17] and thus not comparable; c) although the number of capillary profiles on a histological section has been proved to be a prognostic marker of many types of tumours $[4,7,8,21]$, it is not representative for the factors needed for tumour progression (for example the quantity of the blood circulating in the tumour or the surface provided for proliferation of the tumour cells, etc.) and it depends on the tortuosity of vessels [2]. Our present approach to the problem, which is the assessment of microvascular surface density (outer surface of microvessels in unit tumour volume) and microvascular volume density (volume of microvessels in unit tumour volume) by electron microscopic morphometry [20], eliminates the aforementioned problems.

\section{MATERIALS AND METHODS}

\section{Animals and treatments}

Five-week-old male Wistar rats (Humán Co., Gödöllő, Hungary) were kept on standard laboratory diet (Charles River Hungary Ltd., Budapest, Hungary) for 2 weeks, and then the animals were divided into three groups. First group (12 rats) was given $30 \mathrm{mg}$ per body weight azaserine, an initiator of pancreatic carcinogenesis [22], by an i.p. injection of the aqueous solution (Sigma-Aldrich Ltd., Budapest, Hungary), and changed to semisynthetic diet containing $25 \%$ (w/w) raw soya flour which promotes the development of pancreatic cancer [12]. The vitamin and salt premixes 
(BIO-SERV Co., Holton Industries, Frenchtown, NJ, USA) needed for the semisynthetic diet were prepared according to the recommendation of the American Institute of Nutrition [1]. The other two groups (3-3 rats) were control groups, these animals did not receive azaserine-injection and from the age of seven weeks they were kept on the aforementioned raw soya flour-based diet (soya control) or remained on the standard laboratory diet (normal control). Food and water were available for the animals ad libitum throughout the experimental period. Four rats of azaserine-treated group and all rats of the control groups were sacrificed by decapitation 6 months after the carcinogen initiation and 4-4 azaserine-treated rats were killed by the same way 15 and 20 months after the injection. The pancreas was removed from the animals and processed for electron microscopy.

\section{Morphological and morphometrical methods}

Tissue pieces were fixed in sodium cacodylate-HCl-buffered $(\mathrm{pH}=7.2) 1 \%$ glutaraldehyde, post-fixed in cacodylate-buffered $1 \%$ osmium tetroxide solution, contrasted in 2\% aqueous uranyl acetate and embedded in Durcupan (Fluka Chemie AG, Buchs, Switzerland). Semithin sections were made from the tissue blocks and they were stained with toluidine blue-Azur II with the purpose of morphological investigations. This staining proved to be useful also for detecting the small premalignant lesions (AACNs) in the samples taken 6 months after the carcinogen initiation. In the older animals there were bigger tumours (adenomas or adenocarcinomas) visible to the naked eye which were fixed and embedded separately from the tumour-bearing host tissue (host control). In case of each control group (normal control, soya control and host control) and each tumour stage (6,15 or 20 months after the azaserine-injection) 12-12 tissue blocks were chosen for ultrathin sectioning. Five electron micrographs per sample were taken at a primary magnification of $1400 \times$ by the use of a Jeol JEM-100 CX II electron microscope operated at $60 \mathrm{kV}$. On micrographs with final magnification of $4200 \times$ morphometrical measurements were carried out by Weibel's point counting method [20], using a grid consisting of 1350 points in quadratic arrangement. The microvascular surface density $\left(S_{V}\right)$ and volume density $\left(V_{V}\right)$ were determined according to the next equations described by Weibel [20]:

$$
S_{V}=\frac{2 \times I}{L}\left[\frac{\mathrm{mm}^{2}}{\mathrm{~mm}^{3}}\right] \text { and } V_{V}=\frac{A_{m v}}{A_{t}}\left[\frac{\mathrm{mm}^{3}}{\mathrm{~mm}^{3}}\right] \text { where }
$$

$I \quad$ is the number of intersections between test lines and outlines of microvessels

$L \quad$ is the length of test lines enclosed in the test area altogether

$A_{m v}$ is the number of test points enclosed in the area of microvessel profiles

$A_{t} \quad$ is the total number of test points enclosed in the test area 
The test area was the whole section of exocrine pancreas, and in Fig. $1 S_{V}$ was expressed as $1 / \mathrm{mm}$ and $V_{V}$ as percentage. From data of the 60 micrographs the means for $S_{V}$ and $V_{V}$ were counted for each experimental group. The Mann-Whitney $u$-test, a nonparametric alternative to the $t$-test, was used for the statistical evaluation of the results, because the primary data did not show normal distribution.

\section{RESULTS}

\section{Histological investigations}

On the basis of semithin sections, morphology of the samples taken from normal control, soya control or host control is very similar and shows the typical features of normal pancreas. Six months after the azaserine-injection only small premalignant lesions can be seen under light microscope in the pancreas. These AACNs consist of enlarged cells with decreased basophilia, increased nuclear size and hypertrophic zymogen zone [15]. Fifteen months after the initiation adenomas with diameter of 3-5 $\mathrm{mm}$ can even be found which still have acinar structure and often a thin connective tissue capsule, too. By month 20 adenocarcinomas appear which are even larger and show disintegration of the acinar structure and anaplasia [18]. Since signs of invasion could not be found, we termed this type of tumour localised anaplastic adenocarcinoma.

\section{Quantification of tumour vascularity}

Microvascular density cannot be easily estimated in semithin or histological sections of pancreas, because it is not possible to recognize all capillaries due to their very small size. Their diameter is usually comparable to that of the nucleus of an acinar cell. Using electron micrographs we avoided this difficulty and the determined microvascular surface density $\left(S_{V}\right)$ and volume density $\left(V_{V}\right)$ values are presented in Fig. 1. The results indicate that there is no significant difference between the appropriate $S_{V}$ and $V_{V}$ values of the 3 control groups. Therefore, we correlated the different tumour stages only with the host control. Six months after the carcinogen initiation $S_{V}$ and $V_{V}$ decreased, but not significantly, compared to the host control and until the 15th month both of them remained at the same level. By the 20th month $S_{V}$ and $V_{V}$ increased significantly as compared to the 15 th month. In spite of the observed changes in the degree of vascularity during the tumour progression, only one of the $S_{V}$ or $V_{V}$ values of the tumour stages proved to differ significantly from the results obtained in case of the host control. This can be explained with the very large standard deviations originating from the difference between animals or tumours, respectively. 


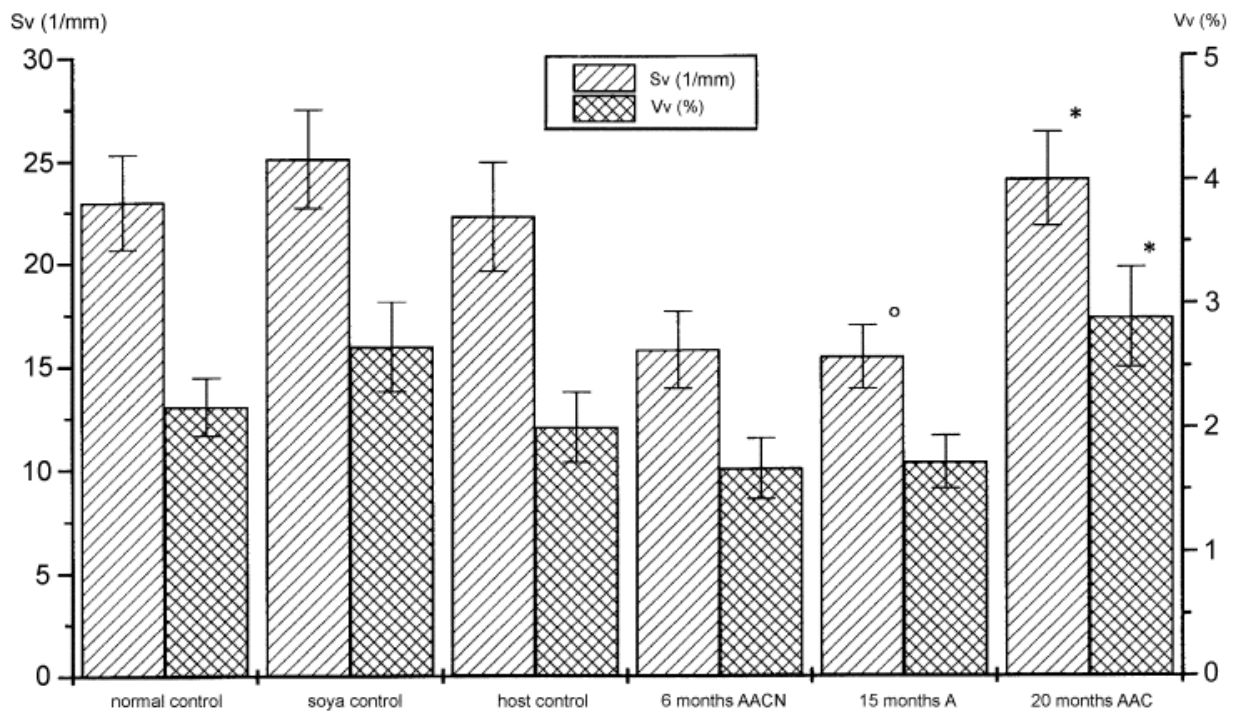

Fig. 1. Microvascular surface density (Sv) and microvascular volume density (Vv) values in control tissues and tumour stages at given time-periods after the azaserine-injection. AACN - atypical acinar cell nodule, A - adenoma, AAC - anaplastic adenocarcinoma. Data are represented by arithmetic mean and SEM (error bar) of 60 values. ${ }^{\circ}$ significant difference compared with host control $(\mathrm{p}<0.05),{ }^{*}$ significant difference compared with previous tumour stage $(\mathrm{p}<0.05)$

\section{DISCUSSION}

The changes in $S_{V}$ and $V_{V}$ are in good correlation with each other except in case of the tumour stage 15th month when $S_{V}$ significantly decreased but $V_{V}$ remained unchanged compared to the values of 6th month. It is conceivable that the average diameter of the microvessels varied between the two aforementioned stages. The fact that there is no significant difference in the examined parameters of vascularity between the control groups suggests that raw soya diet and azaserine do not influence the angiogenesis. In the beginning of the tumour progression (6th and 15th month) $S_{V}$ and $V_{V}$ definitely decreased and remained unchanged at least until month 15 . In other words, vascularization of the premalignant stages was of the same degree indicating a similar rate of tumour and microvessel growth. At some time period after month 15 this equilibrium must have shifted towards microvessel growth leading to an increased vascularity of the month 20 anaplastic adenocarcinomas. Our preliminary results seem to support the angiogenic switch theory of malignisation [9]. It is generally accepted now, that the induction of some angiogenic factors is initiated by hypoxia [16]. Oxygen deficit itself, however may slow down tumour cell growth in larger tumours $[5,13]$, which also may explain in part of the increased share of microvessels from tissue volume in our adenocarcinomas. Quantitation of the rate of 
proliferating endothelial and tumour cells in our samples is needed to confirm this explanation.

Comparison of our results with those of other laboratories involves difficulties since our method for the assessment of tumour vascularity is entirely dissimilar to the usual counting of microvessels, nevertheless, it has many advantages. Using electron microscope we could avoid the uncertainty in recognition of capillaries which emerges in case of light microscopic immunohistochemistry due to the small vessel size and imperfect staining. In addition, morphometric measurement of microvascular surface and volume density is more reliable and results in a more exact quantification of microvessels compared to counting them on hot spots. Estimation of vascular surface density (VSD) under light microscope in different tumour stages was published in cases of prostatic and renal cell carcinomas $[2,10]$ where samples with 3 various degrees of differentiation were compared. VSD in the control tissue proved to be lower than in the 2 tumour grade with higher degree of differentiation but it was as large as or higher than in the third (less differentiated) tumour grade. Relative capillary volume (corresponding to volume density of capillaries) was evaluated using light microscope in moderately and poorly differentiated carcinomas of the esophagus [14]. It was significantly lower in carcinomas than in normal tissue and showed no correlation with tumour progression. These results are partly in contrast with ours and besides these experiments there are essentially no other data, to our knowledge, about measurements and comparison of vascular surface or volume density in different tumour stages. Consequently, further investigations should be performed to clarify the contradictions. Examination of new time points in our model system will contribute to a deeper understanding quantitative microvascular changes during tumour progression.

\section{ACKNOWLEDGEMENTS}

We would like to thank Sarolta Pálfia, Ágnes Keserú and Mariann Saródy for their excellent technical assistance. This study was partly supported by a Copernicus Contract (CIP-ACT 930210) of the European Union.

\section{REFERENCES}

1. American Institute of Nutrition (1977) Report of the American Institute of Nutrition ad hoc committee on standards for nutritional studies. J. Nutr. 107, 1340-1348.

2. Barth, P. J., Weingärtner, K., Köhler, H. H., Bittinger, A. (1996) Assessment of the vascularization in prostatic carcinoma: A morphometric investigation. Hum. Pathol. 27, 1306-1310.

3. Beliën, J. A., Somi, S., de Jong, J. S., van Diest, P. J., Baak, J. P. (1999) Fully automated microvessel counting and hot spot selection by image processing of whole tumour sections in invasive breast cancer. J. Clin. Pathol. 52:3, 184-192.

4. Bochner, B. H., Cote, R. J., Weidner, N., Groshen, S., Chen, S. C., Skinner, D. G., Nichols, P. W. (1995) Angiogenesis in bladder cancer: relationship between microvessel density and tumour prognosis. J. Natl. Cancer Inst. 87:21, 1603-1612. 
5. De Jaeger, K., Merlo, F. M., Kavanagh, M. C., Fyles, A. W., Hedley, D., Hill, R. P. (1998) Heterogeneity of tumour oxygenation: relationship to tumour necrosis, tumour size, and metastasis. Int. J. Radiat. Oncol. Biol. Phys. 42:4, 717-721.

6. Folkman, J. (1985) Tumor angiogenesis. Adv. Cancer Res. 43, 175-203.

7. Fontanini, G., Lucchi, M., Vignati, S., Mussi, A., Ciardiello, F., De Laurentiis, M., De Placido, S., Basolo, F., Angeletti, C. A., Bevilacqua, G. (1997) Angiogenesis as a prognostic indicator of survival in non-small-cell lung carcinoma: a prospective study. J. Natl. Cancer Inst. 89:12, 881-886.

8. Gasparini, G., Weidner, N., Maluta, S., Pozza, F., Boracchi, P., Mezzetti, M., Testolin, A., Bevilacqua, P. (1993) Intratumoral microvessel density and p53 protein: correlation with metastasis in head and neck squamous-cell carcinoma. Int. J. Cancer 55:5, 739-744.

9. Hanahan, D., Folkman, J. (1996) Patterns and emerging mechanisms of the angiogenic switch during tumorigenesis. Cell 86, 353-364.

10. Köhler, H. H., Barth, P. J., Siebel, A., Gerharz, E. W., Bittinger, A. (1996) Quantitative assessment of vascular surface density in renal cell carcinomas. Br. J. Urol. 42:4, 717-721.

11. Longnecker, D. S. (1987) The azaserine-induced model of pancreatic carcinogenesis in rats. In: Scarpelli, D. G., Reddy, J. K. and Longnecker, D. S. (eds): Experimental pancreatic carcinogenesis. CRC Press, Boca Raton. pp. 117-132.

12. McGuinness, E. E., Hopwood, D., Wormsley, K. G. (1982) Further studies of the effects of raw soya flour on the rat pancreas. Scand. J. Gastroenterol. 17, 273-277.

13. Milgross, C. G., Tucker, S. L., Mason, K. A., Hunter, N. R., Peters, L. J., Milas, L. (1997) The effect of tumor size on necrosis and polarographically measured pO2. Acta Oncol. 36:2, 183-189.

14. Porschen, R., Classen, S., Piontek, M., Borchard, F. (1994) Vascularization of carcinomas of the esophagus and its correlation with tumor proliferation. Cancer Res. 54:2, 587-591.

15. Réz, G., Tóth, S., Pálfia, Z. (1999) Cellular autophagic capacity is highly increased in azaserineinduced premalignant atypical acinar nodule cells. Carcinogenesis 20, 1893-1898.

16. Rockwell, S., Knisley, J. P. S. (1997) Hypoxia and angiogenesis in experimental tumor models: Therapeutic implications. In Goldberg, I. D. and Rosen, E. M. (eds): Regulation of angiogenesis. Birkhäuser Verlag, Basel. pp. 335-360.

17. Tanigawa, N., Lu, C., Mitsui, T., Miura, S. (1997) Quantitation of sinusoid-like vessels in hepatocellular carcinoma: its clinical and prognostic significance. Hepathology 26:5, 1216-1223.

18. Tóth, S., Nagy, K., Pálfia, Z., Réz, G. Regulation of cellular autophagic capacity: data from the azaserine-induced pancreatic tumor progression (in preparation)

19. Vermeulen, P. B., Libura, M., Libura, J., O’Neill, P. J., van Dam, P., van Marck, E., van Oosterom, A. T., Dirix, L. Y. (1997) Influence of investigator experience and microscopic field size on microvessel density in node-negative breast carcinoma. Breast Cancer Res. Treat. 42:2, 165-172.

20. Weibel, E. R. (1969) Stereological principles for morphometry in electron microscopic cytology. Int. Rev. Cytol. 26, 235-302.

21. Weidner, N., Folkman, J., Pozza, F., Bevilacqua, P., Allred, E. N., Moore, D. H., Meli, S., Gasparini, G. (1992) Tumour angiogenesis: a new significant and independent prognostic indicator in earlystage breast carcinoma. J. Natl. Cancer Inst. 84:24, 1875-1887.

22. Yager, J. D., Roebuck, B. D., Zurlo, J., Longnecker, D. S., Weselcouch, E. O., Wilpone, S. A. (1981) A single-dose protocol for azaserine initiation of pancreatic carcinogenesis in the rat. Int. J. Cancer 28, 601-606. 